\title{
Physical Activity During the COVID-19 Pandemic in People with Severe Mental Disorders: An Overview of the Portuguese Reality
}

\section{Atividade Física Durante a Pandemia de COVID-19 em \\ Pessoas com Doença Mental Grave: Perspetiva Sobre a Realidade Portuguesa}

Raquel COSTA $\triangle 1$, Tânia BASTOS², Eluana GOMES ${ }^{1}$, Rui CORREDEIRA ${ }^{1}$

Acta Med Port 2021 Sep;34(9):569-571 - https://doi.org/10.20344/amp.16270

Keywords: COVID-19/psychology; Exercise; Mental Disorders; Portugal

Palavras-chave: COVID-19/psicologia; Exercício; Perturbações Mentais; Portugal

Coronavirus disease 2019 (COVID-19) is a public health emergency of international concern that has led to a worldwide crisis with dramatic consequences for life, health, economy, and society. ${ }^{1}$ In Portugal, like in many other countries around the world, the strategies to manage the pandemic have mostly revolved around imposing restrictions. In particular, the social distancing measures and lockdown requirements resulted, among others, in the temporary closing of many crowded environments including fitness centres, public gyms and sports facilities. Albeit essential to decrease the spread of the virus, these measures reduced the number of opportunities to remain physically active, and probably led to a decrease in the physical activity (Physical Activity is defined as any bodily movement produced by skeletal muscles that results in energy expenditure. Physical activity in daily life can be categorized into occupational, sports, conditioning, household, or other activities) levels, while the amount of time spent in sedentary behaviours (e.g., time spending seated or laid down, and watching TV) potentially increased, as described elsewhere., ${ }^{2,3}$ Moreover, the COVID-19 pandemic has posed major challenges for maintaining mental health. For instance, both individuals with and without mental disorders may experience negative psychological effects like distress, and symptoms of depression and anxiety. ${ }^{1,4}$ Although the impact of COVID-19 on the physical activity in those without pre-existing psychiatric disorders has been covered extensively, there has been a limited focus on individuals with severe mental disorders (such as bipolar disorder, schizophrenia, and other psychosis) and emerging psychopathology. This is particularly disconcerting because individuals with severe mental disorders already tend to have low levels of physical activity and present higher risk of infection and of suffering complications from COVID-19 compared to those without a psychiatric disorder, due to the disorder per se, comorbid conditions (obesity, diabetes, hypertension) and cognitive deficits. ${ }^{2,5}$ Moreover, with lockdown restrictions, individuals with severe mental disorders can be more susceptible to stress, and consequently, relapses or worsening of the disease course can occur. $2,6,7$ Sole et $a^{2}$ evaluated the effects of the COVID-19 pandemic and lockdown measures between community controls and patients with mental disorder during the state of emergency and found that patients reported engaging in less physical activity compared with the community controls. Additionally, the study also revealed that during the lockdown, symptoms of anxiety and depression, as well as weight gain, sleep changes and tobacco consumption were more prevalent in the mental disorder group compared to community controls. Nonetheless, this vulnerable population relies on psychiatric services, such as institutions, programs, or group support to encourage and promote a healthy lifestyle including physical active behaviour. ${ }^{8}$ Unfortunately, due to the burden imposed by the pandemic, namely during the period of the state of emergency, the Portuguese National Health System had to adjust in order to ensure the response to essential care needs and the Portuguese Psychiatric and Mental Health units were included in this adjustment - limiting appointments and home visits to the most urgent cases; non-urgent outpatient appointments were carried out by telephone. ${ }^{9}$ Moreover, the temporary closure of several ambulatory facilities for psychiatric patients, including acute day hospitals, led to an abrupt interruption in the provision of ambulatory mental health care to many patients. ${ }^{9}$

In this scenario, it is crucial that government, psychiatric services, and healthcare providers understand the reality of

1. Research Centre in Physical Activity, Health and Leisure (CIAFEL). Faculty of Sport. University of Porto. Porto. Portugal.

2. Centre of Research, Education, Innovation and Intervention in Sport. Faculty of Sport. University of Porto. Porto. Portugal.

$\triangle$ Autor correspondente: Raquel Costa. raquelfcosta7@gmail.com

Recebido: 26 de março de 2021 - Aceite: 28 de julho de 2021 - Online issue published: 01 de setembro de 2021

Copyright $\odot$ Ordem dos Médicos 2021 
people with severe mental disorders in order to support their needs with adequate policies, such as ensuring the continuity of necessary community-based social and healthcare services and providing access to important information. A recent study ${ }^{10}$ demonstrated that meeting physical activity guidelines was strongly associated with a reduced risk for severe COVID-19 outcomes among infected adults. Therefore, the promotion of physical activity should be prioritised by public health agencies and incorporated into routine medical care. ${ }^{10}$ Due to the vulnerability of people with severe mental disorders ${ }^{5}$ it is crucial to improve their physical activity levels by implementing innovative and effective strategies.

Firstly, it is crucial to understand that people with severe mental disorders often exhibit limited motivation to engage in physical activity and to maintain an active lifestyle. ${ }^{11-13}$ Several factors can explain this behaviour, namely, the presence of negative and depressive symptoms, low selfefficacy and lack of confidence and somatic co-morbidities associated with the disorder (e.g., metabolic syndrome and clinical pain). ${ }^{11-13}$ Therefore, the implementation of motivational strategies is essential to engage this population in physical activity. Strategies such as a relationship of trust with mental healthcare professionals, positive interactions with peers, and engaging in physical activity in a non-clinical environment are linked with starting and maintaining physically active behaviours. ${ }^{12,13}$ However, due to the COVID-19 pandemic, all these motivational processes became difficult to enforce. Therefore, an adjustment to the conventional approach became necessary. For example, during telephone appointments, online psychoeducation programs or online mindfulness-based interventions, mental healthcare professionals supported by qualified exercise professionals, could spend a few minutes to discuss the details of a healthy lifestyle and encourage patients to perform more physical activity. Some strategies can be suggested as discussed below.

\section{Increasing physical activity levels}

Costa et $\mathrm{al}^{11}$, in a Portuguese sample of patients with schizophrenia, demonstrated that autonomous motivation (i.e., when patients behave with a full sense of volition and choice) is a significant predictor of physical activity. The authors suggested that clinical practice guidelines should highlight the importance of autonomous motivation to improve attitudes towards physical activity. Evidence revealed that in order to increase the level of autonomous motivation, clinicians should minimize pressure, adopt supportive language, and promote pleasant activities in a positive environment. ${ }^{12,13}$ For example, qualified exercise professionals with expertise in exercise (Exercise is a subset of physical activity that is planned, structured, and repetitive and has as a final or an intermediate objective the improvement or maintenance of physical fitness) prescription, and additional knowledge regarding psychopathology, can help patients create a new weekly schedule that should be both enjoyable and feasible. Due to the pandemic, the regular daily routines have changed, including the routine to engage in physical activity. Therefore, a new and personalized schedule is important to provide some structure to individuals with severe mental disorders. Qualified exercise professionals can provide support and guidance to create the schedule, for instance, by suggesting when to include the exercise sessions, the duration of each session, what type of activity (e.g., walking, yoga, circuit training), clarify what will be the necessary equipment (if applicable), and the intensity (i.e., low, moderate, and high). It is important to consider the physical activity recommendations from the World Health Organization ${ }^{14}$ : adults should do at least 150-300 minutes of moderate-intensity aerobic physical activity; or at least 75-150 minutes of vigorous-intensity aerobic physical activity throughout the week. Combinations of moderate and vigorous intensity exercise can be performed in order to meet this recommendation. Adults should also do musclestrengthening activities at moderate intensity or greater that involve all major muscle groups on two or more days a week. In order to help achieve these recommendations, workout resources (e.g., exercise illustrations or exercise videos) should be provided. These can be facilitated by online services or, if this is not possible, copies or flyers with exercise illustrations (e.g., Manual de Boas Práticas em Atividade Física na Doença Mental) $)^{15}$ can be sent by mail.

\section{Decreasing sedentary behaviour}

A systematic review and meta-analysis ${ }^{16}$ analysed sedentary time of people with psychosis and the authors found that this population spent more than 11 hours of their waking day being sedentary. These high levels of sedentary behaviour, along with the decline of cardiovascular health in this population, highlights the need for approaches specifically targeting sedentary behaviour. ${ }^{16}$ The World Health Organization ${ }^{14}$ recommends that adults should limit the amount of time spent being sedentary. In order to reduce sedentary time, it is important to avoid long periods of inactivity - for example by using external cues such as advert breaks or set alarms to perform a movement break (e.g., stretching, five jumps, walking to the door and returning, climbing stairs) when watching TV. In the same way, performing daily activities like cleaning and cooking, gardening, and farming; performing a short exercise workout (e.g., relaxation exercises, abs, stretching, strength condition using body weight and/ or objects in the house [e.g., chairs, water bottles, rice package]) are also good strategies to reduce sedentary time and consequently improve physical activity 
levels. Walking is also a good activity to increase physical activity levels (but not enough to meet the above physical activity recommendations), for example people with severe mental disorder should be encouraged to choose walking to the nearest supermarket instead of using a car or public transport.

Furthermore, the use of devices and social support could also be good strategies to maintain physical activity levels and decrease sedentary behaviour in people with severe mental disorders. There is evidence demonstrating that mental health technologies for activity tracking are feasible and acceptable for use among patients with severe mental disorders. ${ }^{17}$ Apps and devices - for example activity monitors, set with reminders to move, can track activity levels and step counts. Mental healthcare professionals can motivate each person to increase their physical activity level every day, for example by increasing the number of steps or reduce the time spent in sedentary behaviours. It is important to take into consideration that this vulnerable population can present significant difficulties in terms of access to technologies or resources that are commonly used by the general population. Regarding social support, a recent systematic review ${ }^{18}$ showed that family members play a pivotal role in supporting health parameters, like cardiovascular care of people with severe mental disorders. Therefore,

\section{REFERENCES}

1. Holmes EA, O'Connor RC, Perry VH, Tracey I, Wessely S, Arseneault L, et al. Multidisciplinary research priorities for the COVID-19 pandemic: a call for action for mental health science. Lancet Psychiatry. 2020;7:54760.

2. Sole B, Verdolini N, Amoretti S, Montejo L, Rosa AR, Hogg B, et al. Effects of the COVID-19 pandemic and lockdown in Spain: comparison between community controls and patients with a psychiatric disorder. Preliminary results from the BRIS-MHC STUDY. J Affect Disord. 2021;281:13-23.

3. Stockwell S, Trott M, Tully M, Shin J, Barnett Y, Butler L, et al. Changes in physical activity and sedentary behaviours from before to during the COVID-19 pandemic lockdown: a systematic review. BMJ Open Sport Exerc. 2021;7:1-7

4. Kontoangelos K, Economou M, Papageorgiou C. Mental Health Effects of COVID-19 Pandemia: A Review of Clinical and Psychological Traits. Psychiatry Investig. 2020;17:491-505.

5. Yao H, Chen JH, Xu YF. Patients with mental health disorders in the COVID-19 epidemic. Lancet Psychiatry. 2020;7:e21.

6. Kozloff N, Mulsant BH, Stergiopoulos V, Voineskos AN. The COVID-19 Global Pandemic: Implications for People With Schizophrenia and Related Disorders. Schizophr Bull. 2020;46:752-7.

7. Yocum AK, Zhai Y, McInnis MG, Han P. Covid-19 pandemic and lockdown impacts: A description in a longitudinal study of bipolar disorder. J Affect Disord. 2021;282:1226-33.

8. Muruganandam P, Neelamegam S, Menon V, Alexander J, Chaturvedi SK. COVID-19 and Severe Mental Illness: Impact on patients and its relation with their awareness about COVID-19. Psychiatry Res. 2020;291:113265.

9. Goncalves-Pinho M, Mota P, Ribeiro J, Macedo S, Freitas A. The Impact of COVID-19 Pandemic on Psychiatric Emergency Department Visits - A Descriptive Study. Psychiatr Q. 2020:1-11

10. Sallis R, Young DR, Tartof SY, Sallis JF, Sall J, Li Q, et al. Physical mental healthcare professionals can encourage the household to adopt a healthy lifestyle as well - sharing periods of physical activity with others in the household can be more motivating to people with severe mental disorders. Finally, social support will be beneficial for both physical and mental health.

These strategies, along with a prompt and effective response in the other domains of the patient's life (e.g., treatment engagement, family support, social inclusion), holds the potential to support people with mental disorders to maintain physical active behaviours throughout the pandemic, promoting physical and mental well-being and preventing the widening of health inequalities. ${ }^{19}$

\section{AUTHORS CONTRIBUTION}

RC: Drafted the manuscript.

TB, EG: Provided input and critical revision.

RC: Reviewed the manuscript.

\section{COMPETING INTERESTS}

The authors declare they have no conflicts of interest.

\section{FUNDING SOURCES}

No funding was available for the elaboration of this manuscript.

inactivity is associated with a higher risk for severe COVID-19 outcomes: a study in 48440 adult patients. Br J Sports Med. 2021(in press). doi: 10.1136/bjsports-2021-104080.

11. Costa R, Bastos T, Probst M, Seabra A, Vilhena E, Corredeira R. Autonomous motivation and quality of life as predictors of physical activity in patients with schizophrenia. Int J Psychiatry Clin Pract. 2018;22:184-90.

12. Farholm A, Sorensen M. Motivation for physical activity and exercise in severe mental illness: A systematic review of intervention studies. Int J Ment Health Nurs. 2016;25:194-205.

13. Vancampfort D, De Hert M, Stubbs B, Ward PB, Rosenbaum S, Soundy $A$, et al. Negative symptoms are associated with lower autonomous motivation towards physical activity in people with schizophrenia. Compr Psychiatry. 2015;56:128-32.

14. World Health Organization. WHO guidelines on physical activity and sedentary behaviour. Geneva: WHO; 2020.

15. Bastos T, Costa R, Gomes E, Pizarro A, Corredeira R. Manual de Boas Práticas em Atividade Física na Doença Mental. Porto: Faculdade de Desporto da Universidade do Porto; 2019.

16. Stubbs B, Williams J, Gaughran F, Craig T. How sedentary are people with psychosis? A systematic review and meta-analysis. Schizophr Res. 2016;171:103-9.

17. Naslund JA, Aschbrenner KA, Barre LK, Bartels SJ. Feasibility of Popular m-Health Technologies for Activity Tracking Among Individuals with Serious Mental Illness. Telemed E-Health. 2015;21:213-6.

18. Zabeen S, Phua D, Mohammadi L, Lawn S. Family involvement to support cardiovascular self-management care for people with severe mental illness: a systematic review. J. Ment. Health. 2020 (in press). doi: 10.1080/09638237.2020.1818194.

19. Campion J, Javed A, Sartorius N, Marmot M. Addressing the public mental health challenge of COVID-19. Lancet Psychiatry. 2020;7:657-9. 